\title{
APPROXIMATE APPROACH OF RESEARCH AND ASSESSMENT OF CRACK RESISTANCE OF CYLINDRICAL HOUSINGS
}

\author{
Volodymyr Bulgakov $^{1}$, Aivars Aboltins ${ }^{2}$, Oleksii Kutsenko ${ }^{3}$, Semjons Ivanovs ${ }^{2}$, Simone Pascuzzi ${ }^{4}$ \\ ${ }^{1}$ National University of Life and Environmental Sciences of Ukraine, Ukraine; \\ ${ }^{2}$ Latvia University of Life Sciences and Technologies, Latvia; \\ ${ }^{3}$ Taras Shevchenko National University of Kyiv, Ukraine; ${ }^{4}$ University of Bari Aldo Moro, Italy \\ semjons@apollo.lv
}

\begin{abstract}
In this work, we propose a procedure that allows us to quickly, without involving finite-element packages at the execution stage, evaluate the state of postulated cracks in cylindrical structural elements with internal anticorrosion cladding under pressure and unsteady temperature loading. The procedure contains three components. The first component of the procedure is based on the analytical solution of the unsteady thermal conductivity problem. The second component of the procedure is also analytical relations specifying circumferential and longitudinal stresses in a two-layer cylinder as a function of internal pressure and temperature distribution along the depth of the wall. In the third stage of the procedure, the coefficients of stress intensity along the crack front are determined by the method of influence functions using the known distribution of stresses. To implement this part of the procedure, the influence functions must first be calculated by the finite element method. In this work the example of calculating the functions of influence for longitudinal semi-elliptical cracks of 1/8 wall thickness depth and with the ratio of the principal half-diameters 0.3 and 0.7 is presented. As an example of the implementation of the procedure, comparative calculations were carried out and the elastic-plastic calculation of a cylindrical shell containing longitudinal cracks was given. Stress intensity coefficients along the part of the front located in the base metal were calculated. The results of analytical calculations were conservative until plastic deformations developed in the cladding. At significant plastic strains, the values of the stress intensity coefficients calculated by the proposed procedure, on the contrary, are significantly inferior. This demonstrates once again the fact that calculations in the framework of linear fracture mechanics are not always conservative in relation to calculations in the elastic-plastic formulation. The paper gives an explanation of the reason for the obtained effect.
\end{abstract}

Keywords: resistance, thermal shock, fast, cylinder.

\section{Introduction}

When investigating the crack resistance of equipment, it is often necessary to quickly estimate the level of values of stress intensity coefficients corresponding to the considered loading scenario. There is a sufficient number of reference books on fracture mechanics, containing tabulated or approximated dependences of stress intensity coefficients on the stresses in the crack location area. Therefore, the main problem in finding the values of the stress intensity coefficients is the determination of the corresponding stresses. Even in the case of simple equipment geometries, such as a cylindrical vessel or a rectilinear section of a pipeline, the distribution of these stresses over the wall thickness may be complex in the case of nonstationary temperature loading. The problem becomes more complex if the cylinder wall is composed of two layers of different materials. Double-layered cylindrical structural elements are often found in technological equipment, the inner surface of which is protected by anticorrosion cladding.

In [1-4] an algorithm for the construction of so-called $p-T$ curves. In this case, simple scenarios of changing the coolant temperature at a constant conservative value of the heat exchange coefficient are used as the load. The latter circumstance significantly reduces the area of application of the algorithm proposed in [1], since in real scenarios, the heat transfer coefficient between the coolant and the cylinder wall changes in wide ranges. The algorithm [1] in this work is generalized to the case of arbitrary change in the heat transfer coefficient in time. This generalization makes it possible to carry out fast crack resistance estimations for real scenarios of changes in thermal-hydraulic parameters. In spite of the fact that in real scenarios the coolant temperature distribution is not always axisymmetrical, the proposed approach has the right to exist. It is known [5] that under the conditions of a transient process, the solution of the axisymmetric thermoelasticity problem allows us to approximate well the maximum values of axial and circumferential stresses arising in the wall of a cylindrical vessel under a load that is non-uniform in circumferential direction. In this case, the values of circumferential stresses are even somewhat more conservative.

The article [6] presents a completely analytical solution of the problem of the growth of an annular crack in a solid homogeneous cylinder under the action of an unsteady heat load. The article [7] offers 
a solution of a stationary thermoelastic problem for a two-layer cylindrical inclusion with a radial crack. However, these papers are dealing with endless cracks, which are rarely met in practice.

Relatively close to this study is paper [8]. However, in this work the temperature and stress fields in the wall of a two-layer cylinder are obtained numerically, using the finite element method. Therefore, the originality of the proposed work lies in the combination of analytical methods of calculation, which make it possible to determine the stress intensity factors along a crack in the wall of a two-layer cylinder, subjected to a non-stationary heat load.

The proposed algorithm implies the sequential performance of three steps: determination of the unsteady temperature field along the wall thickness of a bilayer cylinder, based on the distribution of temperature and internal pressure values, calculation of circumferential and longitudinal stresses, and calculation of stress intensity factors along the front of longitudinal cracks by circumferential stresses and stress intensity factors along the front of transverse cracks by longitudinal stresses. The peculiarities of each of the stages are discussed below.

\section{Materials and methods}

In the case of small wall thickness of a vessel or a pipeline with respect to its radius, the corresponding axisymmetric heat conduction problem can be approximated by the problem for a rod. In [1] the solution of such a problem is given under the condition of homogeneity of initial temperature distribution. This solution cannot be used directly for temperature calculation in loading scenarios, where the heat transfer coefficient changes in time. For this purpose, it should be supplemented by the solution of the problem with homogeneous boundary conditions, but with an arbitrary initial temperature distribution. The solution of the latter problem can be represented as a superposition on eigenfunctions of the corresponding problem on eigenvalues $\mu_{n}$ and eigenfunctions $X_{n}(x)$ :

$$
T(x, t)=\sum_{n=1}^{\infty} a_{n} X_{n}(x) e^{-\mu_{n}^{2} \omega t},
$$

where

$$
X_{n}(x)=\left\{\begin{array}{cc}
\Delta_{1}\left(\mu_{n}, x\right), & 0<x<h_{1}, \\
\Delta_{2}\left(\mu_{n}, x\right), & h_{1}<x<h=h_{1}+h_{2},
\end{array} \quad a_{n}=\frac{\int_{0}^{h} T_{0}(x) X_{n}(x) d x}{\int_{0}^{h} X_{n}^{2}(x) d x}, n=1,2, \ldots,\right.
$$

$t$ - time;

$x$ - axial coordinate of the rod;

$T_{0}(x)$ - initial temperature distribution along the rod;

$h_{1}$ and $h_{2}$ - lengths of the homogeneous parts of the rod.

Expressions for functions $\Delta_{1,2}\left(\mu_{n}, x\right)$ and parameter $\omega$, as well as the characteristic equation with respect to $\mu_{n}$, listed in [1].

The superposition of (1) and the solution given in [1] is a solution of the heat conduction problem for an arbitrary initial temperature distribution over the wall of a bilayer cylinder and a constant value of the heat transfer coefficient between the inner surface of the cylinder and the liquid. Let us call this problem a basic one. The solution of the basic problem also makes it possible to find the temperature in the cylinder at an arbitrary change in the heat exchange coefficient over time. For this purpose, the entire time interval of the transient process is divided into subintervals, at which the heat transfer coefficient changes little and can be considered constant - averaged over the subinterval. Also in each subinterval, the fluid temperature change should be well approximated by a linear function, which can always be achieved by selecting time subintervals small enough. After splitting into subintervals, the general thermal conductivity problem is decomposed into a sequence of basic problems of the same type. In this case, the initial temperature distribution at each interval after the first one is obtained as the final distribution at the previous subinterval. The second component of the procedure are the expressions for the circumferential $\sigma_{\varphi}$ and longitudinal $\sigma_{Z}$ stresses in a bilayer cylinder caused by temperature and internal pressure not uniformly distributed over the wall thickness. The corresponding expressions are given in [4]. With some corrections, they can also be taken from [9]. The above relations specify the solution of the axisymmetric thermoelasticity problem for a bilayer cylinder when the internal pressure and temperature distribution over the thickness of its wall are known. 
The third component of the procedure is the method of finding the stress intensity coefficients according to the known stress distributions - the method of influence functions. According to it, the stress intensity coefficients at each point of the crack front $K_{I}$ are represented as a simple sum:

$$
K_{I}\left(s_{m}\right)=\sum_{n=1}^{N} b_{n} K_{I n}\left(s_{m}\right), m=1,2, \ldots, M .
$$

where $s_{m}, m=1,2, \ldots, M-$ points along the crack front, in which the values of the stress intensity coefficients are determined;

$K_{I n}$ - impact functions,

$b_{n}$ - coefficients, which are determined by the least-squares method from the condition of the best approximation of the normal stresses on the crack banks:

$$
\sigma(\bar{x}) \approx \sum_{n=0}^{N} b_{n} f_{n}(\bar{x}),
$$

where $f_{n}(\bar{x})$ - basis functions on which the decomposition is carried out.

Fig. 1. shows schematic images of sub-surface and surface semi-elliptical cracks of depth $a$ and length $2 c$. Local coordinate axes $\bar{x}$ and $\bar{y}$ are directed along the main diameters of the corresponding ellipses. As a rule, the stress distributions varying in the normalized degrees of the local depth coordinate are chosen as basis functions:

$$
f_{n}(\bar{x})=\left(\frac{\bar{x}}{a}\right)^{n}, 0 \leq \bar{x} \leq a, n=0,1, \ldots, N .
$$

The value of $N$ in (3) depends on how much the pressure on the fracture banks changes with depth. In practice, in most cases, it is possible to limit the value to $N=1$ or $N=2$.
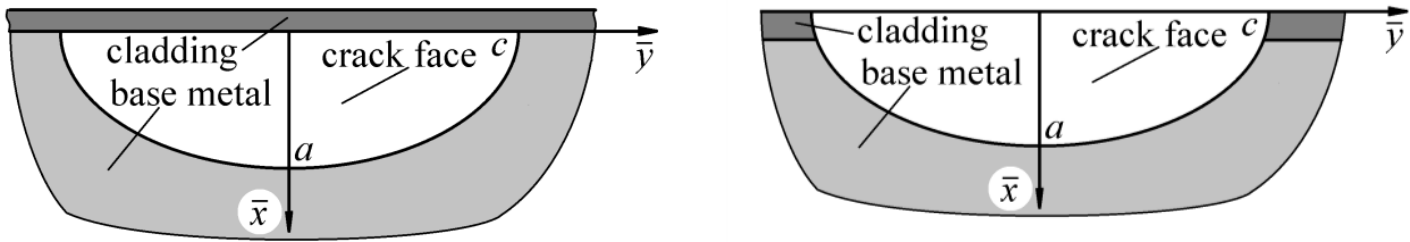

Fig. 1. Undercladding (left) and surface (right) semielliptical cracks

Fig. 2. and Fig. 3. show the first three influence functions $(n=0,1,2)$ for sub-slab semi-elliptic cracks with the ratio of principal half-diameters $a / c=0.7$ and $a / c=0.3$. Crack depth $a$ is $25 \mathrm{~mm}$, which is $1 / 8$ of the cylinder wall thickness $h=h_{1}+h_{2}$. Cladding thickness $h_{1}$ selected to be $7 \mathrm{~mm}$. The influence functions were calculated using the finite element package CalculiX [10]. Figures 4 and 5 show the result of calculating the normal displacements of the crack banks for the first influence function $(n=0)$. Stress intensity coefficients of the influence functions were determined by extrapolating normal displacements in the vicinity of the crack front.

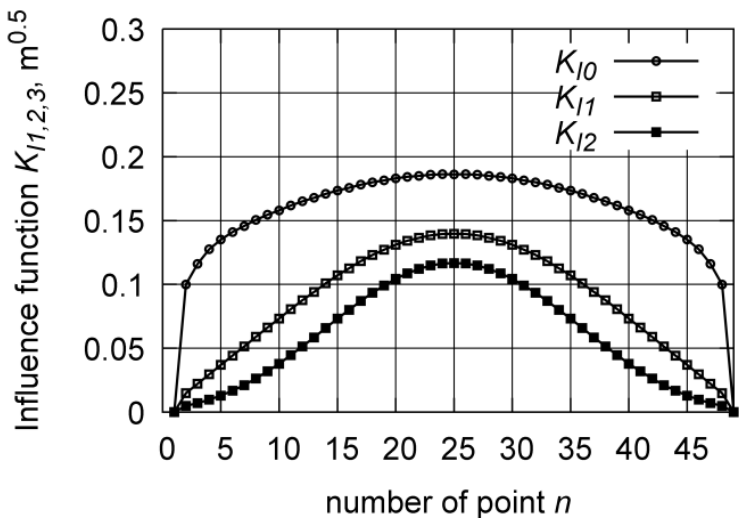

Fig. 2. Influence function for crack with $a / c=0.7$

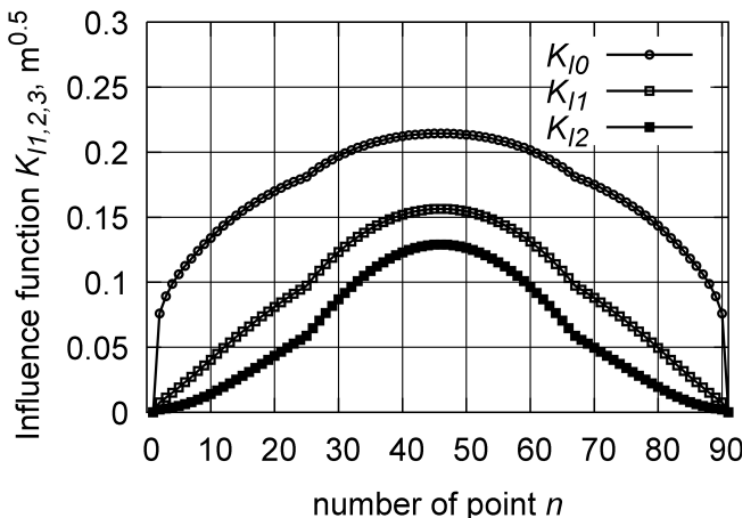

Fig. 3. Influence function for crack with $a / c=0.3$ 

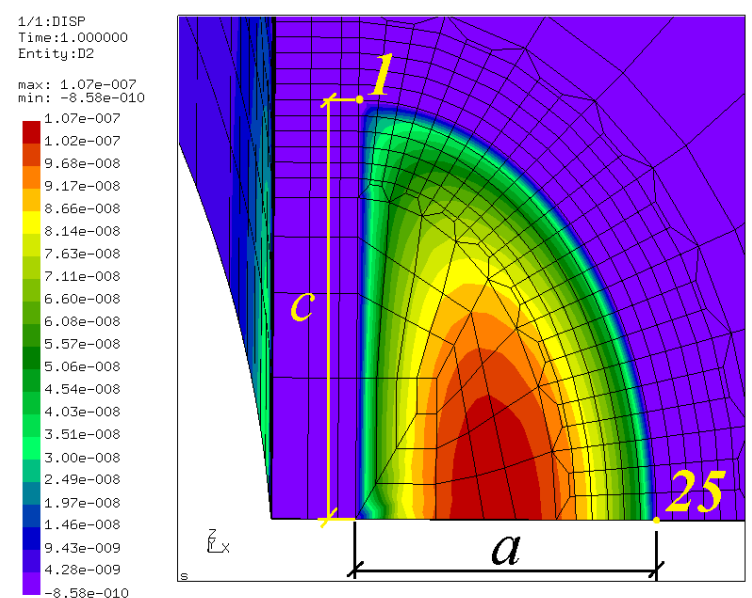

Fig. 4. Normal displacements of face points of crack with $a / c=0.7$

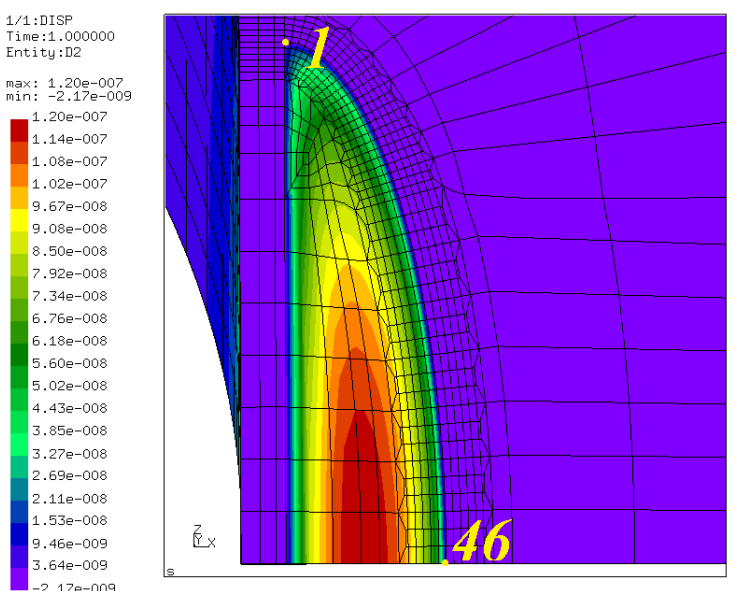

Fig. 5. Normal displacements of face points of crack with $a / c=0.3$

\section{Results and discussion}

The limits of this approach were considered using the model scenario of thermal shock of a cylindrical hull given in [2]. This scenario assumes complete (axisymmetric) cooling of the case. Change of thermal hydraulic parameters: coolant temperature $T$, heat transfer coefficient $H$ and internal pressure $p$ see Fig. 6 . The dots on the graphs indicate values at key points in time, at time intervals between them all thermal-hydraulic parameters change linearly in time.

The calculations were performed for the geometric parameters of the two-layer cylinder corresponding to the cylindrical shell of the cylindrical body: inner radius $r_{1}=2068 \mathrm{~mm}$, surfacing thickness $h_{1}=7 \mathrm{~mm}$, base metal thickness $h_{2}=192.5 \mathrm{~mm}$. The thermal and mechanical properties of the materials were taken from [2].

Two calculations of temperatures and circumferential stresses were performed. One calculation was based on analytical relations. Let us call it "Analytic". The second calculation was carried out numerically by the finite element method using the package CalculiX. This calculation will be referred to as "FEM". The need for this calculation is dictated by the limited data given in [2]. In addition, the calculations in [2] were performed in the elastic-plastic formulation on models with embedded cracks, which makes it impossible to estimate the stresses in the cylinder without cracks. When carrying out analytical calculations, the physical properties of the materials were taken as averaged, while the numerical calculations were temperature-dependent.

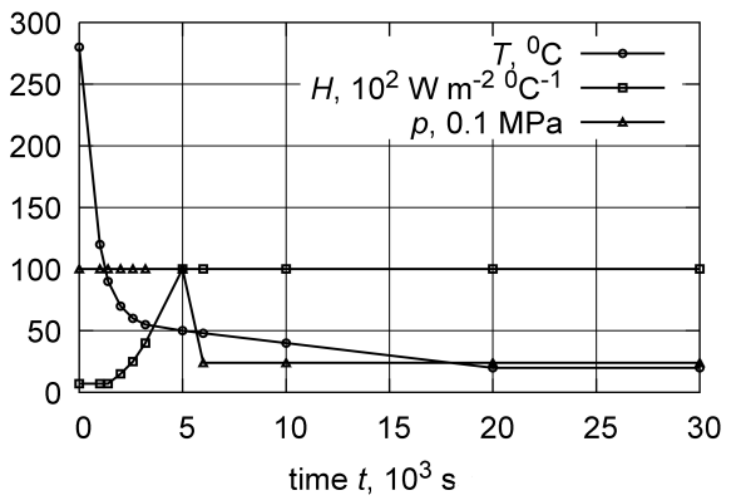

Fig. 6. Thermalhydraulics parameters of scenario

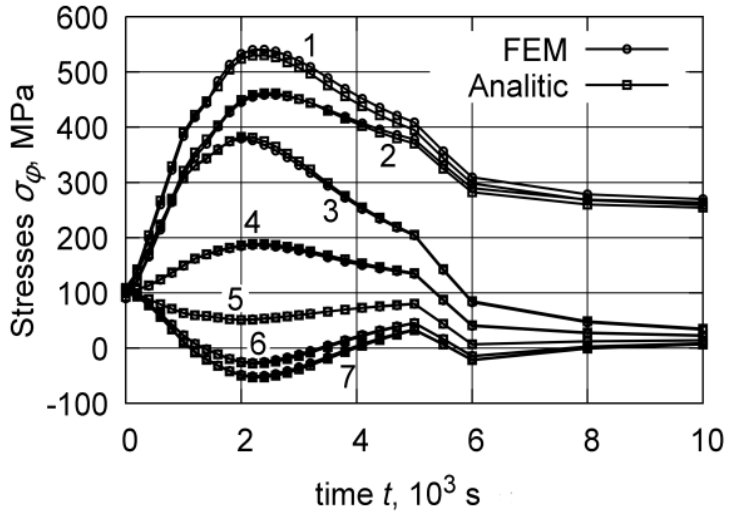

Fig. 7. Stresses evolution during first 10000 s obtained from different calculations 
The results of analytical and numerical calculations of circumferential stresses in comparison are shown in Figure 8. The time dependences are presented for seven points spaced along the wall thickness: from point 1 on the inner surface of the cylinder to point 7 on the outer surface of the cylinder. It can be seen that all the plots are grouped in pairs into seven consecutive families of curves depending on the calculated point. In each of the families it is practically impossible to distinguish the plots within their accuracy. The relative error of the analytical calculations of both circumferential stresses and temperatures does not exceed $3 \%$.

Results of comparison of stress intensity coefficient distributions along the crack front with the ratio of principal half-diameters $a / c=0.7$ different moments of the scenario are shown in Fig. 8-11 in comparison with the numerical calculation. The results of the numerical calculations are taken from the calculations carried out within the framework of [2]. Recall that in [2] the calculations were performed in the elastic-plastic formulation. Along the crack front, the $J$-integral was calculated and the equivalent SIC value was determined from its value. When performing analytical calculations, the error of approximation of circumferential stresses on the fracture banks by three basis functions according to formula (2) did not exceed $0.01 \%$.

As it follows from Fig. 8, at the initial moment of time the stress intensity coefficient calculated by the proposed procedure has slightly higher values. This is about the same at the time of 200 seconds (see Fig. 9). But already at $t=400$ seconds the situation changes dramatically: the numerical and analytical values approximately coincide (Fig. 10). Later, the values of the stress intensity coefficients calculated numerically begin to significantly exceed those obtained analytically. This is evidenced by the graphs shown in Fig. 11, which show the distribution of the stress intensity coefficients at a finite moment of time. Similar behavior is demonstrated by the calculation results for the crack with the ratio of the principal half-diameters $a / c=0.3$.

The conclusions are also confirmed by the graphs in Fig. 12 and Fig. 13, which show the evolution over time of the stress intensity coefficients at the deepest points of both cracks. Note that in the numerical calculation, the deepest point ceases to be the most dangerous from a certain point, as the values of the stress intensity coefficients at the points near the interface rapidly increase.

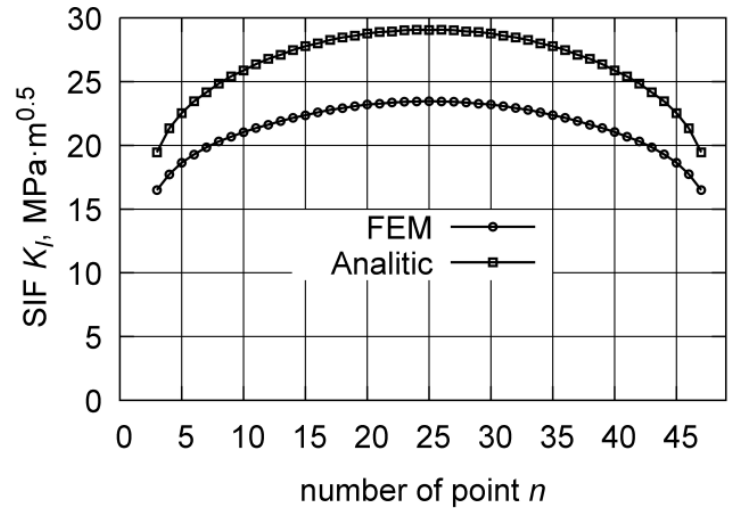

Fig. 8. SIF distribution at time $\boldsymbol{t}=\mathbf{0} \mathrm{s}$ for crack with $a / c=0.7$

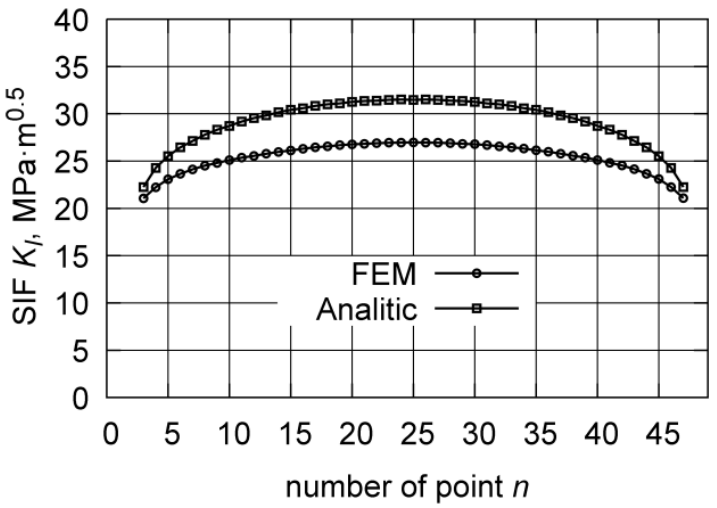

Fig. 9. SIF distribution at time $\boldsymbol{t}=\mathbf{2 0 0 \mathrm { s } \text { for }}$ crack with $a / c=0.3$

In order to make sense of the results obtained, it is important to remember that the proposed analytical procedure assumes linear-elastic behavior of the materials. Numerical calculations were carried out taking into account the plastic properties, first of all, the plastic properties of the cladding. At the temperatures in question, the cladding material begins to show significant plastic properties at stresses of about 150 to $200 \mathrm{MPa}$. If we refer to Fig. 7 (curves 1 and 2), we can conclude that such stress values are reached in the cladding by about 200 seconds. Later on, the cladding can no longer be considered linearly elastic, which is confirmed by the results of numerical calculations. The cladding becomes more malleable and it further loads the crack front points located in the base metal, especially near the cladding. As a consequence, as plastic deformation increases in the cladding, the values of stress intensity coefficients along the crack front level off. After the maximum load passes in the points of the front near the interface, the stress intensity coefficient becomes even higher than at deeper ones, which 
is explained by the effect of the base metal wedging by the cladding. This effect is discussed in detail in [2]. All the mentioned features are deprived of the solution obtained by the proposed procedure. The main difference of this solution is the failure to take into account the plastic properties of the cladding, which leads to a significantly lower level of stress intensity coefficients compared to the numerical solution after the stresses in the cladding reach the limit of proportionality.

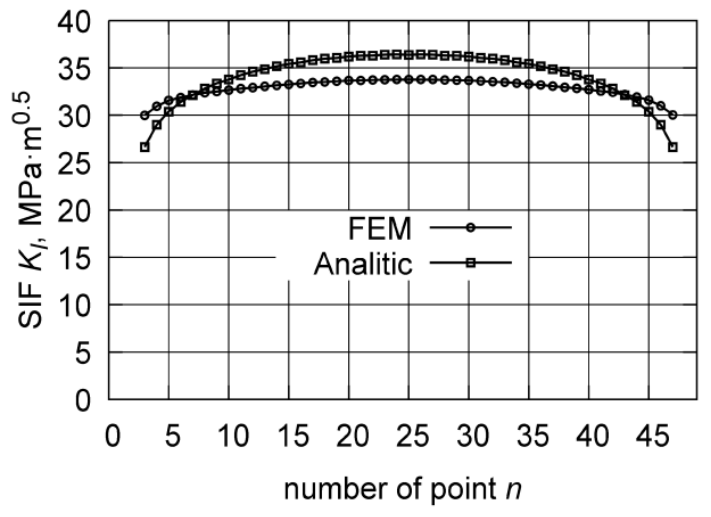

Fig. 10. SIF distribution at time $t=400 \mathrm{~s}$ for crack with $a / c=0.7$

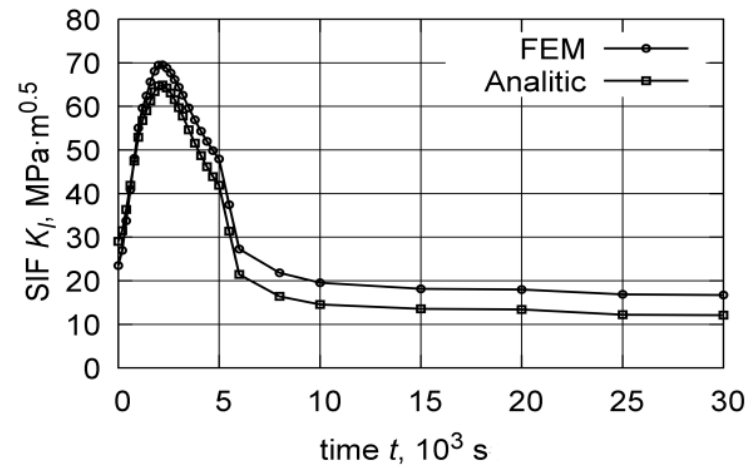

Fig. 12. SIF evolution at deepest point of crack with $a / c=0.7$

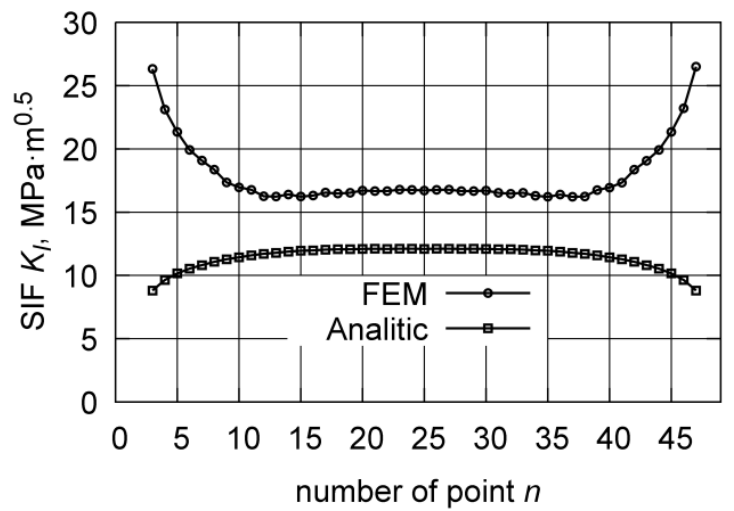

Fig. 11. SIF distribution at time $t=30000 \mathrm{~s}$ for crack with $a / c=0.3$

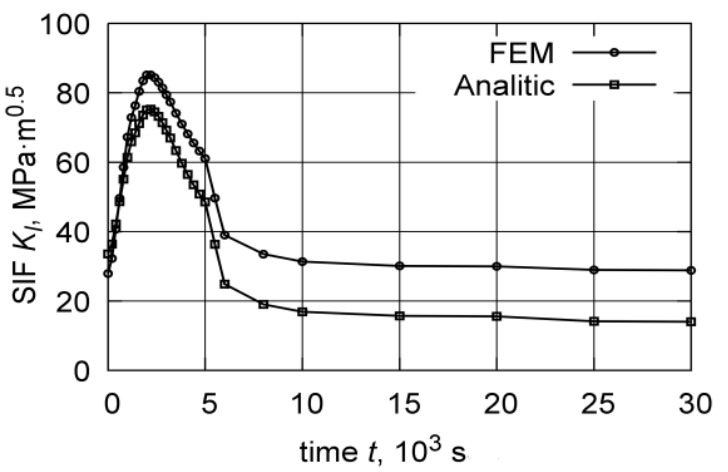

Fig. 13. SIF evolution at deepest point of crack with $a / c=0.3$

The obtained results are in qualitative agreement with the results of the other studies. For example, the distributions of circumferential stresses at fixed points in time, shown in Fig. 3 in the work [8], coincide by their nature with those obtained by us, but not presented here in order to save space (they can be approximately restored on the basis of the data in Fig. 8). In addition, it is difficult to speak of a quantitative coincidence, since in [8] another scenario of thermal shock is considered, the initial data of which are unknown to us. The same applies to other similar studies. That is why the work [2] was chosen for comparison since the script and other parameters are given in it in full.

\section{Conclusions}

The proposed procedure makes it possible to adequately calculate unsteady axisymmetric fields of temperatures and stresses in the walls of rectilinear sections of pipelines or vessels, the inner surface of which is coated with anticorrosion cladding. The computational relationships are simple in nature, since only elementary functions are used. Based on the method of influence functions, the procedure on the calculated stress fields allows to find distributions of stress intensity coefficients in postulated cracks.

The accuracy of the proposed approach was investigated on the example of longitudinal semielliptical cracks in the wall of the cylindrical part of the body. Comparison with the numerical elasticplastic calculation showed that: 
- provided that the stresses in the wall, which does not contain a crack, do not exceed the material proportionality limit, the calculation of the stress intensity coefficients along the front of the underlaying cracks can be carried out according to the proposed procedure as conservative;

- in case the limit of material proportionality is exceeded, the calculation of stress intensity coefficients along the front of underlaying cracks cannot be carried out according to the proposed procedure, since it leads to underestimated results.

Since sub-surface cracks are usually small in size, significant stresses must develop in the material in order to reach critical values of the stress intensity coefficients at the points of their fronts. This limits the application of the proposed procedure in particular and of linear mechanics methods in general to the case of surface cracks.

\section{References}

[1] Kadenko I., Sakhno N., Kutzenko O., Kharytonov O. An Analytical Approach to the Generation of the Operational Limit Curves with NDE-based Crack Postulation. Proc. 8h Intl. Conf. on NDE in Relation to Structural Integrity for Nuclear and Pressurised Components, Berlin, 2010, pp. 234242.

[2] Kutsenko O., Kadenko I., Pitoiset X., Kharytonov O.M., Sakhno N., Kravchenko I. Effect of neutron irradiation hardening of the base metal on the results of WWER-1000 reactor pressure vessel residual lifetime assessment. Int. J. Press. Vessel. Pip., Vol. 171, 2019, pp. 173-183.

[3] Murakami, Y. (ed.), Stress Intensity Factors Handbook. In 2 Volumes. Oxford etc. Pergamon press, $1987,1456 \mathrm{pp}$.

[4] Kutsenko O., Kadenko I., Kharytonov O., Sakhno N. An analytical basis for the generation of NPP emergency operation limiting pressure-temperature curves. Mathematical Modeling And Computing, Vol. 3, №1, 2016, pp. 79-89.

[5] Pressurized Thermal Shock in Nuclear Power Plants: Good Practices for Assessment (IAEA TECDOC 1627). Vienna: IAEA, 2010, $217 \mathrm{p}$.

[6] Zhornik V.A., Prokopenko Yu.A., Rybinskaya A.A., Savochka P.A. Ring-shaped crack propagation in a cylinder under nonsteady cooling. High Performance Structures and Materials III.- WIT Press Southampton U.K., Boston USA, 2006, pp. 521-526.

[7] Zelenyak V. M. Thermoelastic interaction of a two-component circular inclusion with a crack in the plate. Materials Science. Vol. 48 (3), 2012, pp. 301-307.

[8] Kharchenko V. V., Kobel'skii S. V., Kravchenko V. I., Chirkov A. Yu., Zvyagintseva A. A. Determination of Stress Intensity Factor for Semielliptical Face Cracks in a Body of WWER-1000 Nuclear Reactor by Results of the Solution of Boundary Problems of Thermoelasticity on the Basis of Mixed FEM Scheme. Problems of Strength. Vo.2, 2007, pp. 45-51.

[9] Rules and Regulations for Design and Stress Analysis for Components and Piping of Nuclear Installation (PNAEG-G7-002-86). Moscow: Energoatomizdat, 1989, 525 p. (In Russian)

[10] Dhondt G. The Finite Element Method for Three-Dimensional Thermomechanical Applications. Hoboken: Wiley, 2004, 340 p. 